\title{
Vom Smart Grid zur Smart Security
}

\author{
H. Leopold OVE
}

Online publiziert am 29. Juni 2013

(C) Springer Verlag Wien 2013

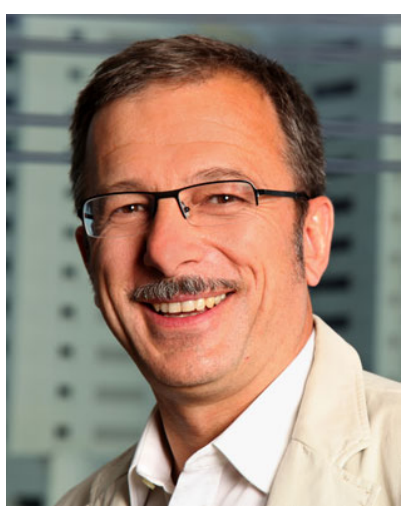

Dipl.-Ing. Helmut Leopold
Die Lösungssuche für unsere großen gesellschaftlichen Fragestellungen zu Energie, Mobilität, Sicherheit, Gesundheit bzw. Betreuung von zunehmend mehr Menschen beim Älterwerden sowie der Wunsch nach mehr persönlicher/individueller Sicherheit hat besonders im Bereich der modernen Informations- und Kommunikationstechnologien (IKT) eine gewaltige Innovationsdynamik ausgelöst.

Wir vernetzen Fahrzeu-

ge, um von intelligenter Verkehrssteuerung zu profitieren, wir vernetzen Energienetze mit Haushaltsgeräten zu zukünftigen Smart Grids, und wir bauen Telemedizinsysteme, um medizinische Versorgungsleistungen einfach und günstig in unsere Haushalte zu bringen bzw., um den wachsenden Ansprüchen der Gesellschaft an eine zukünftige (technisch unterstützte) flexible und einfache medizinische Versorgung und vor allem Vorsorge gerecht zu werden. Machineto-Machine (M2M) Communication ist dabei ein häufig verwendetes Schlagwort, welches diese umfassende IKT-Vernetzung in allen Lebensbereichen bezeichnet.

Vor allem im Energiebereich ist dabei aktuell eine besondere Dynamik zu verzeichnen. Einerseits ist dies durch das Interesse der öffentlichen Hand gegeben, um einen nachhaltigen Beitrag für unsere Energieeffizienzziele zu schaffen. Andererseits werden durch die Verfügbarkeit neuer Informations- und Kommunikationstechnologien neue innovative Ansätze ermöglicht.

Während bislang der Betrieb von modernen Informations- und Kommunikationstechnologien maßgeblich von funktionierenden Energienetzen abhing, wird sich diese Abhängigkeit in Zukunft auch in die Gegenrichtung entwickeln. Die in rasantem Tempo entstehenden Smart Grids werden in den kommenden Jahren in ganz erheblichem Ausmaß an IKT-Systeme gekoppelt sein, und ein Ausfall oder eine Fehlfunktion wird auch die Energieübertragung und Verteilung wesentlich beeinflussen. Wenn dann sogar noch individuelle Steuerungsmodifikationen von den privaten Hauhalten über ein Smart Grid durchgeführt werden, wird die wechselseitige Abhängigkeit von Energie- und IKT-Netz um ein Vielfaches angewachsen sein.

Bis jetzt wurde die Entwicklung von IKT durch die Innovationsdynamik der Entwicklung neuer Funktionalitäten bestimmt. Durch die neue Bedeutung als "Mission Critical"-Infrastruktur für alle Lebensbereiche einer modernen Gesellschaft - also vor allem im Bereich der zukünftigen Smart Grids - haben wir neue Anstrengungen zu unternehmen, um eine fehlende "Safety \& Security" - dies bezieht sich sowohl auf den Schutz von Daten als auch auf die Ausfallssicherheit bzw. Zuverlässigkeit der Systeme - nicht als "Innovationshemmer" oder sogar Bedrohung für die Entwicklung unserer Gesellschaft zu erleben.
Im vorliegenden Themenschwerpunkt dieser e\&i-Ausgabe wird genau diese Problematik der Gewährleistung von Sicherheit im zukünftigen Smart Grid thematisiert; anhand einiger wichtiger Projekte aus dem Forschungs- und Industriebereich werden Einblicke in kommende Anforderungen und zu verfolgende Ansätze gegeben.

Drei große Initiativen der öffentlichen Hand unterstützen dabei die Anstrengungen von Smart Security-Forschungskooperationen: das österreichische Sicherheitsforschungsprogramm KIRAS und das europäische Sicherheitsforschungsprogramm - viele der angeführten Projekte werden durch diese Programme unterstützt - sowie die Koordinierungsinitiative des BMVIT, wie im Interview mit Herrn Hübner in dieser e\&i-Ausgabe dargestellt. Herr Hübner vom BMVIT beschreibt im Gespräch mit der e\&i die Zielsetzungen und Aktivitäten der öffentlichen Hand, um diesen essentiellen Technologiebereich als nationale Technologieinitiative zu gestalten.

In der Rubrik Praxis \& Wissen dieser Ausgabe werden durch Beiträge von Herrn Hammer, BMVIT, sowie von Frau Klonk, FFG, kurz die nationalen und europäischen Forschungsprogramme dargestellt. Weiters werden einzelne Schlüsselthemen von Smart Security Grids erörtert. Die Herren Beirer, GAI NetConsult, Bosin, TIWAG-Netz, und Kasper, RWE Deutschland, fassen in ihrem Artikel die Aktivitäten der Energienetzbetreiber zusammen, um den sicheren Betrieb von Energienetzen zu gewährleisten.

Darüber hinaus finden Sie in dieser Ausgabe folgende Originalarbeiten: Der Beitrag von Herbert Saurugg und Joe Pichlmayr unterstreicht die besondere Problematik der Komplexität, mit der wir vor allem im Bereich der vernetzten Energienetze zunehmend konfrontiert werden. Das Paper von Thomas Bleier fasst jene IKTSicherheitsaspekte zusammen, die bei Smart Grids zu beachten sind, und zeigt existierende Best practice-Beispiele und vorhandene Standards auf.

Der Beitrag von Berthold Haberler, Linz Strom, Georg Kienesberger, TU Wien, sowie von Friederich Kupzog und Lucie Langer, AIT, zeigt die ersten Projektansätze einer systematischen Bewertung der IKT-Sicherheit der diversen Smart Grid-Projekte auf.

Im Beitrag von Andreas Berl et al. von der Universität Passau und Universität Lancaster wird ein Ansatz aus der Telekommunikation die Benutzung von virtuellen Netzdiensten - als zukunftsweisender Ansatz einer flexiblen und kosteneffizienten Technologie zur Steuerung von Smart Grids beschrieben. Abschließend zeigt das Paper von Christian Wagner et al. vom AIT am Beispiel einer IT-Vernetzung von IT-Systemen im E-Government-Bereich, dass innovative Ansätze verfolgt werden können und, dennoch besondere Datenschutzvorgaben sicher gestellt werden können.

Ich hoffe, Ihnen, sehr geehrte Leserinnen und Leser, mit dieser Auswahl von interessanten Beiträgen eine Standortbestimmungen in Sachen intelligenter und zukunftssicherer Smart Security GridTechnologie zu geben sowie einen Beitrag zu einem wachsenden Bewusstsein für diesen hochbrisante Themenbereich zu leisten.

Leopold, Helmut, Dipl.-Ing., Head of Safety \& Security Department am AIT, Präsident der GIT Gesellschaft für Informations- und Kommunikationstechnik im OVE, AIT Austrian Institute of Technology GmbH, Donau-City-Straße 1, 1220 Wien, Österreich (E-Mail: helmut.leopold@ait.ac.at) 
Abschließend möchte ich Sie noch auf die beiden Arbeitskreise in der GIT Gesellschaft für Informations- und Kommunikationstechnik im OVE hinweisen, die zu dem aktuellen Thema der Sicherheit von Energieinformationsnetzen zwei Arbeitskreise unterhält: „Energy goes ICT" unter der Leitung von Johannes Stadler von
Wien Energie sowie "Cyber Security" unter der Leitung von Thomas Bleier vom AIT. Beide Arbeitskreise geben regelmäßig elektronische Newsletter heraus, die Sie bei Interesse gerne kostenlos unter http://git.ove.at/news.html abonnieren können. 